\title{
Simulation of Single-Arm Fractional Spiral Antennas for Millimeter Wave Applications
}

\author{
Kuo-Fong Hung ${ }^{(1)}$ and Yi-Cheng Lin* (2) \\ (1) Graduate Institute of Communication Engineering, National Taiwan University, Taiwan. \\ (2) Department of Electrical Engineering, National Taiwan University, Taiwan \\ *Email: yclin@cc.ee.ntu.edu.tw
}

\begin{abstract}
Summary
In this paper, a planar single-arm fractional spiral antenna for millimeter wave applications is proposed. Outer-fed by microstrip line, the antenna is printed on a planar PCB with a circular aperture on the ground plane. The antenna design is illustrated with a 5 mil-thick RO5880 substrate with a circular aperture of diameter $3.7 \mathrm{~mm}$ operating at $44 \mathrm{GHz}$. Promising results are achieved, including the $10-\mathrm{dB}$ return loss bandwidth of $35 \%$, the $3-\mathrm{dB}$ axial ratio bandwidth around $20 \%$, and the antenna gain of 2.6 to $3.9 \mathrm{dBic}$ in the $40-48 \mathrm{GHz}$ band.
\end{abstract}

\section{Introduction}

The conventional spiral antenna [1] is usually fed at the center by a vertical coaxial line with balun circuits. In general, the feeding networks at the input ends of spiral arms are quite complicated in design for the broadband balanced transition in $3 \mathrm{D}$ structure. As the operation frequency increases, the fabrication work becomes very difficult for the small dimensions near the spiral center. Hence, a spiral antenna with planar outer feeds is desirable, especially for millimeter wave applications. The planar design may also facilitate the antenna integrated with RF components or MMICs on the same printed circuit board (PCB) and further reduce the size and cost of the transceiver module.

Recently, several outer-fed designs of printed spiral antennas were reported in the literature [2-4] and have been employed for full planar applications. However, these designs confronted some obstacles including the narrowband axial ratio and unstable gain variation over the operation band. In addition, they all are based on the Archimedean spiral that requires several turns to achieve sufficient bandwidth of the 3-dB axial ratio. Since the radiating area of high-frequency is dominated by the inner part of the Archimedean spiral, these outer-fed antennas would experience great loss in the higher band due to the long feed path. Therefore, less turns and shorter spirals are the criteria in the outer-fed design. This paper presents a novel design of planar spiral antenna based on the equiangular spiral [1]. Unlike conventional equiangular spirals, the proposed antenna employs a single-arm spiral starting from the outer end with negative spiral rates. The antenna is named 'fractional spiral' in that only fractional turns are needed in the final design. 


\section{Antenna Design and Results}

Figure 1 shows the antenna geometry and the coordinate system. The single-arm fractional spiral antenna is printed on a single-layer PCB substrate of dielectric constant $\varepsilon_{r}$ and thickness $h$. The backed ground plane contains a circular aperture of diameter $\mathrm{D}$ that confines the radiating area and determines the operation frequency. The spiral arm is fed at outer end by a microstrip line with impedance transformed to standard 50-Ohm port. The shape of the equiangular spiral arm is formed by two exponential curves $C_{1}$ and $C_{2}$, of which the polar equations are expressed as $C_{1}: \rho=(D / 2) e^{-a \phi}$ and $C_{2}: \rho=(D / 2-t) e^{-b \phi}$, where $a$ and $b$ are the spiral rate coefficients of $C_{1}$ and $C_{2}$ respectively, and $\mathrm{t}$ is the off-set parameter that determines the initial width of the spiral at the input end. Note that the width of the spiral arm is broadened as the angle $\phi$ increases from 0 (x-axis), implying the spiral arm may extend to certain ended angle, denoted by $\phi_{e}\left(\phi_{e}<2 \pi\right)$. As a result, a spiral arm with fractional turns is formed. For the purpose of millimeter wave applications, the propose antenna is illustrated on a RO5880 substrate of dielectric constant $\varepsilon_{r}=2.2$, loss tangent $\tan \delta=0.002$, and thickness $h=0.125 \mathrm{~mm}$. A square PCB of length $\mathrm{L}=10 \mathrm{~mm}$ is selected and a circular aperture of diameter $\mathrm{D}=3.7 \mathrm{~mm}$ on the ground plane is designed. The other antenna dimensions are optimized as follow: $t=0.05 \mathrm{~mm}, a=0.2, b=0.4$, and $\phi_{e}=1.25 \pi$.

Design and simulation results of the proposed fractional spiral antenna are accomplished with Ansoft HFSS. The simulated input return loss of the proposed antenna is shown in Fig. 2. The 10-dB return loss bandwidth ranges from 36 to $50 \mathrm{GHz}$, more than $30 \%$. Figure 3 shows the radiation patterns of both RHCP and LHCP. It shows the antenna radiates dominant RHCP waves in the upper semi-shpere but LHCP waves in the lower semi-sphere, as expected. Figure 4 shows the frequency response of the antenna gain and the axial ratio as observed at zenith. It shows the $3-\mathrm{dB}$ axial ratio bandwidth of the proposed antenna is about $20 \%$. The RHCP directivity is found stable and ranges from 2.6 to $3.9 \mathrm{dBic}$ in the $40-48 \mathrm{GHz}$ band. In practice, the antenna is backed by a cavity to generate a unidirectional beam normal to the PCB plane.

\section{Acknowledgement}

This work was supported in part by the National Science Council (NSC) of Taiwan, under the contract no. NSC 94-2219-E-002-007 and NSC 94-2752-E002-002-PAE.

\section{References}

[1] Dyson, J., 'The equiangular spiral antenna,' IRE Trans. Antenna and Prop., Vol. 7, No. 2, pp. 181-187, 1959. 
[2] Nakano, H., Hirokawa, G., Yamouchi, J., and Hirose, K., 'Spiral antenna with coplanar strip line feed,' Electron. Lett., Vol. 28, No.23, pp.2130-2131, 1992.

[3] Gschwendtner, E., Loffler, D., and Wiesbeck, W., 'Spiral antenna with external feeding for planar applications,' IEEE AFRICON 1999, Vol. 2, pp. 1011-1014, 1999.

[4] Morimoto, T. and Kawasaki, S., 'A novel laminated printed spiral antenna for higher gain,' Proceedings of APMC 2001, Taipei, Taiwan, pp. 476-479, 2001.

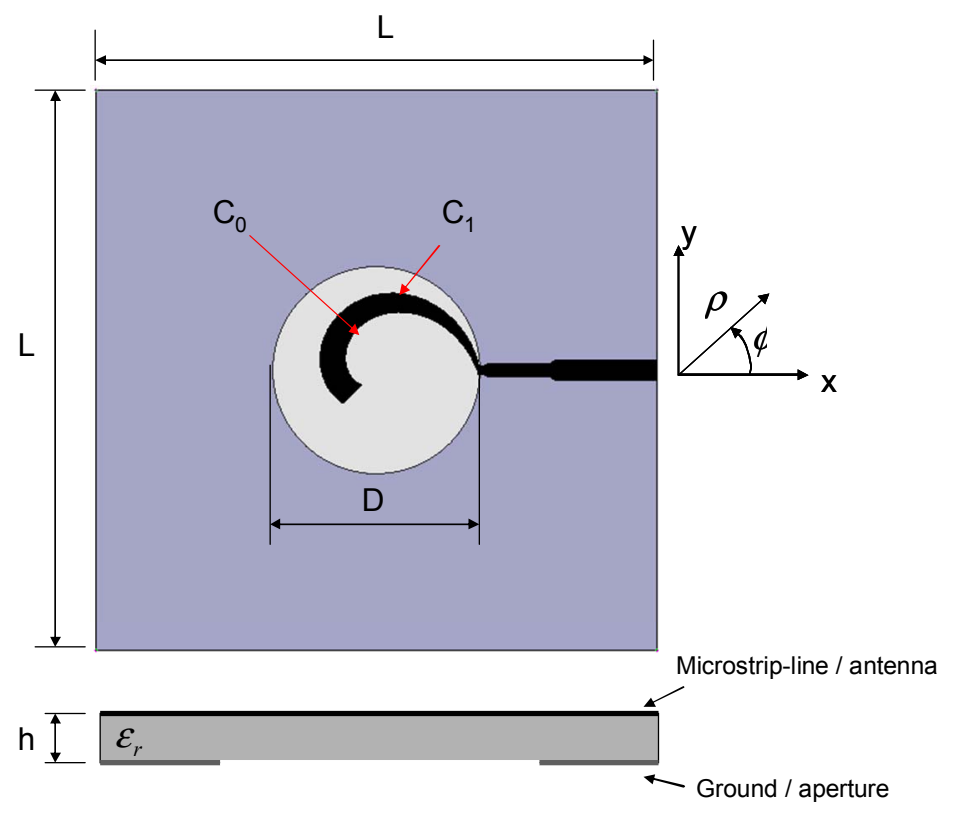

Fig.1. Geometry and coordinate system of the proposed antenna

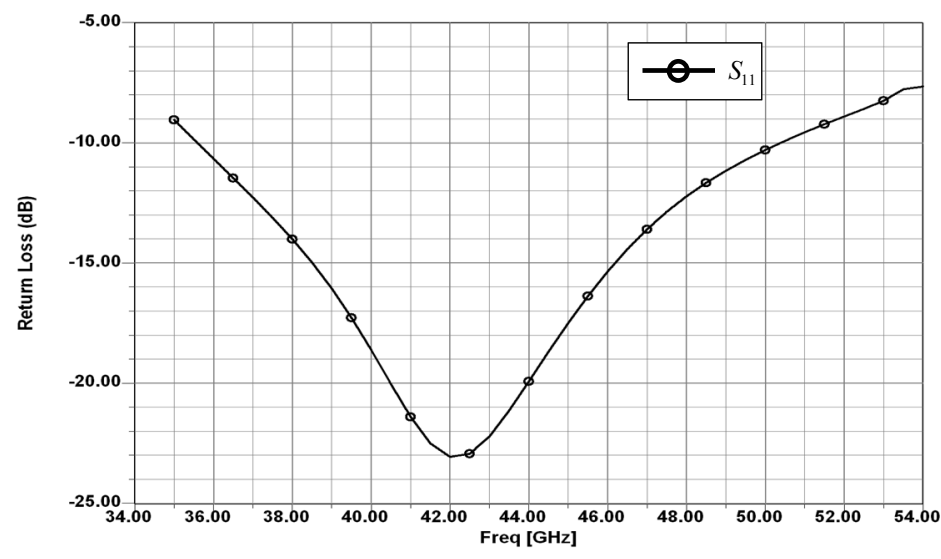

Fig.2. Return loss of the proposed antenna 


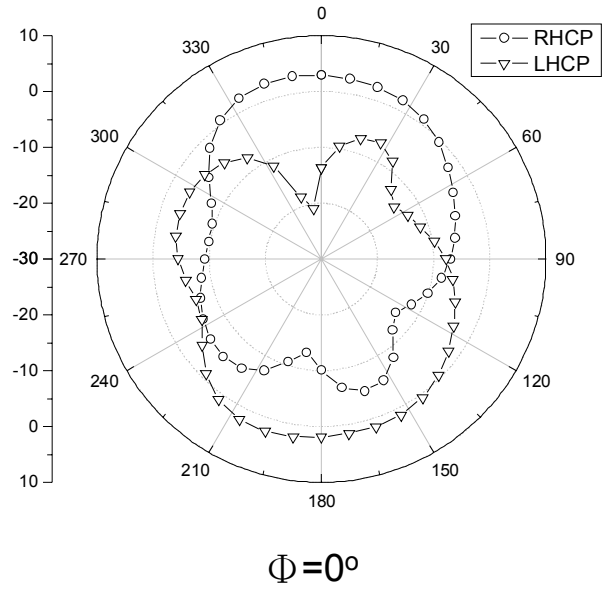

(a)

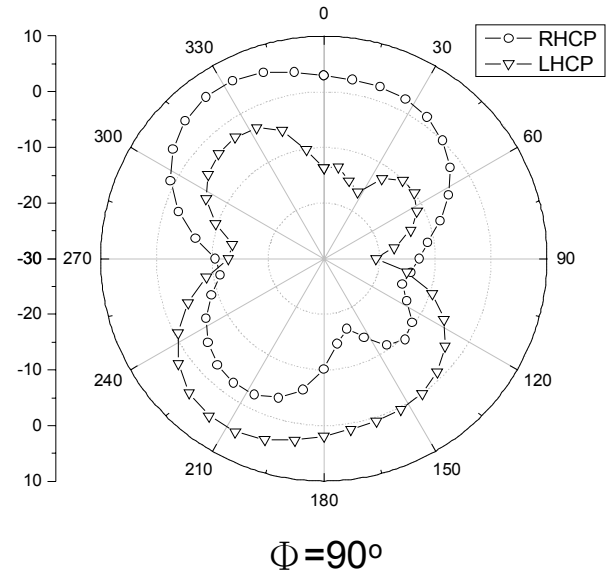

(b)

Fig.3. Polarized patterns (RHCP and LHCP) at $44 \mathrm{GHz}$ in (a) XZ-plane, (b) YZplane

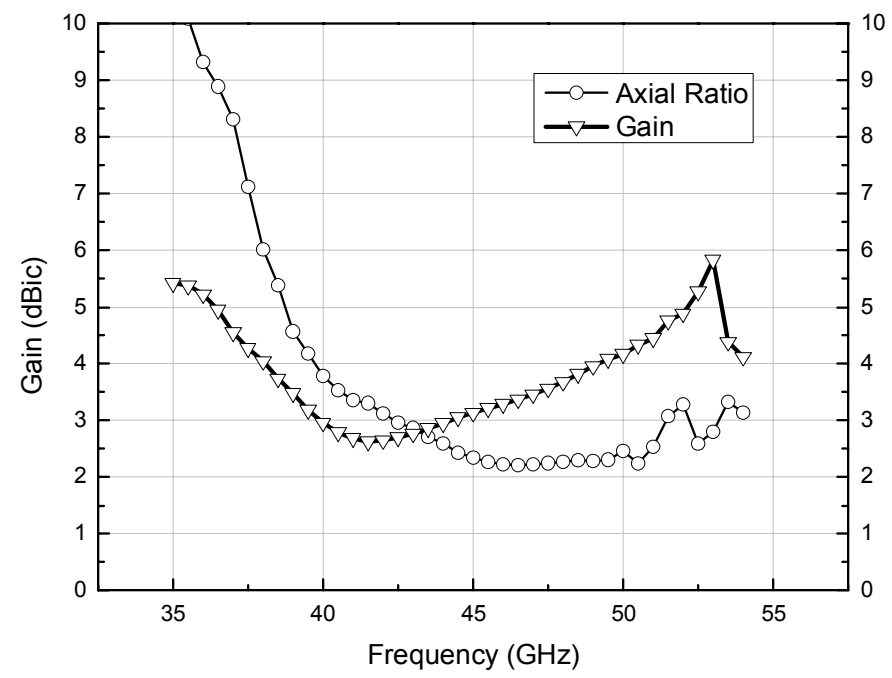

Fig.4. Spectrum of the radiation directivity and axial ratio at zenith ( $\theta=0, \mathrm{z}$-axis) 